\title{
Development of turbulence in submerged jets as a noise-induced transition
}

\author{
P.S. Landa ${ }^{a}$ and P.V.E. McClintock ${ }^{b}$ \\ ${ }^{a}$ Department of Physics, Lomonosov Moscow State University, 119899 Moscow, Russia. \\ ${ }^{b}$ Department of Physics, Lancaster University, Lancaster LA1 4YB, UK.
}

26 February 2004

\begin{abstract}
Experiments show that the amplitude of turbulent pulsation in submerged jets rises with increasing distance from the nozzle, at first slowly and then, after a certain distance, rapidly. This dependence on distance from the nozzle closely resembles the dependence of an order parameter on temperature in the case of a second-order phase transition. Following an idea introduced by Landa and Zaikin in 1996, it is suggested that the onset of turbulence is a noise-induced phase transition similar to that in a pendulum with a randomly vibrated suspension axis. The Krylov-Bogolyubov asymptotic method is used to provide an approximate description of the transition. Results obtained in this way are shown to coincide closely with experimental data. Such an approach is appropriate because the convective character of the instability means that turbulence in nonclosed flows cannot be a selfoscillatory process, as is often assumed. Rather, it must originate in the external random disturbances that are always present in real flows.
\end{abstract}

Keywords: Turbulence, submerged jet, noise, nonequilibrium phase transition

\section{INTRODUCTION}

It is well known that fluid flow in channels is laminar for small flow velocities and turbulent for large flow velocities. ${ }^{1}$ The problem of how turbulence originates has long attracted the considerable attention of researchers. Different scientists responded to this question in different ways. For example, Landau considered the development of turbulence as the excitation of self-oscillations, and he wrote a phenomenological equation similar to the truncated van der Pol equation for the amplitude of self-oscillations in a vacuum tube generator, commenting: ${ }^{2}$ "With further increase of the Reynolds number new periods appear sequentially. As for the newly appeared motions, they have increasingly small scales". As a result, multi-frequency self-oscillations with incommensurate frequencies, i.e. quasi-periodic motion, must set in. Associated with these self-oscillations, there would have to be an attractor in the form of a multi-dimensional torus in the system phase space. Over a wide range of frequencies such quasi-periodic self-oscillations differ little in appearance from chaotic ones, which is why developed turbulence is perceived as a random process.

In the 1970s, after the discovery of deterministic chaos and the realization that a multi-dimensional torus is unstable, ${ }^{3}$ the Landau theory became open to question, but the conception of self-oscillations was retained. The difference lay only in that, instead of quasi-periodic self-oscillations, they became spoken of as chaotic ones. Thus, according to these new ideas, the onset of turbulence is the sudden birth of a strange attractor in the phase space of certain dynamical variables. ${ }^{4}$ We note that similar ideas were repeatedly expressed by Russian scientist Neimark. ${ }^{5}$

We suggest, however, that turbulence arising in nonclosed fluid flows is not a self-oscillatory process. It is known that the instability of such flows is of a convective character, rather than being absolute. This means that a disturbance arising at some point of the flow will not increase indefinitely with time, but will drift downstream. It follows from this property of convectively unstable systems that they are not self-oscillatory, although they are amplifiers of disturbances. For such a system to become self-oscillatory, global feedback must be introduced,

E-mails: landa@phys.msu.su and p.v.e.mcclintock@lancaster.ac.uk 

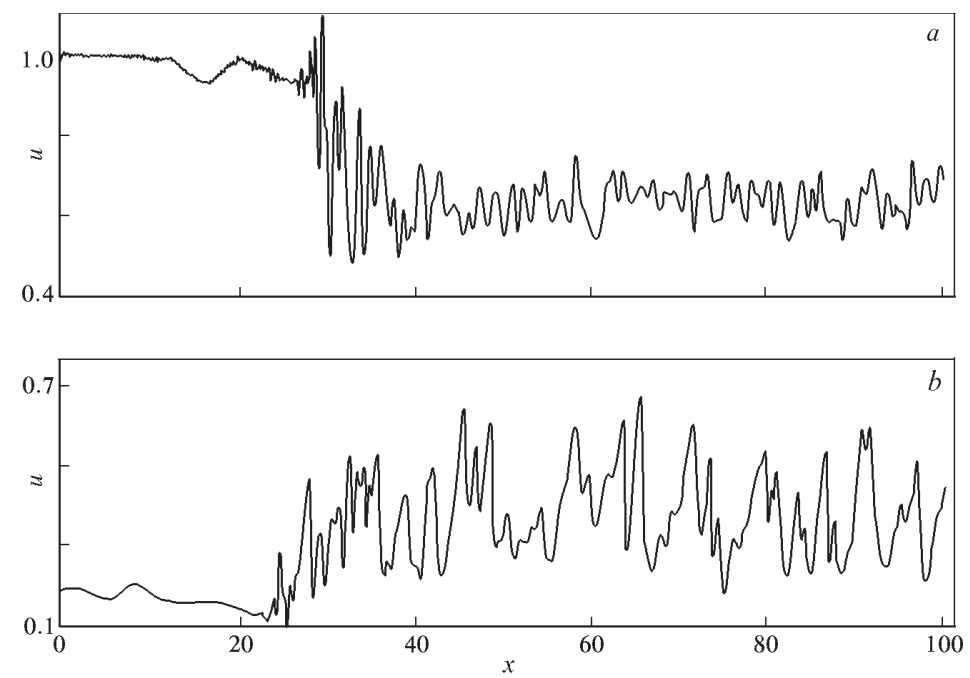

Figure 1. Results of a numerical experiment on pipe flow. Instantaneous distributions of the longitudinal velocity component $u$ in a steady regime for $A / u_{0}=0.04$ are shown: a along the pipe axis $(r / R=0.02)$ and $b$ near the pipe wall $(r / R=0.93)$. After N.V. Nikotin. ${ }^{8}$

e.g. by closing the system in a ring. It should be noted that, in essence, this is exactly what occurs in the process of numerical simulation with periodic boundary conditions. Disturbances are necessarily present in all real systems, both from external sources (environmental fluctuations) and as a result of the molecular structure of a substance (inherent fluctuations). The disturbances can be included as external forces in the equations describing the system behavior. Klimontovich used the fluctuation-dissipation theorem to calculate ${ }^{6}$ the forces caused by the natural fluctuations in hydrodynamic flows. In jet flows the presence of fluctuations, especially at the input, is crucial because they are precisely what lead eventually to the turbulent disturbances observed. It follows from this that an approach to turbulence within the framework of (deterministic) dynamical systems theory is not always appropriate.

\section{FLUID FLOW IN A CIRCULAR PIPE}

One piece of evidence suggesting that turbulence is not a self-oscillatory process comes from the numerical experiments of Nikitin. ${ }^{7,8}$ He simulated fluid flow in a circular pipe of a finite length and radius $R$ with a given velocity at the input cross-section, and with so-called 'soft' boundary conditions at the output cross-section; these latter are

$$
\frac{\partial^{2} u}{\partial x^{2}}=\frac{\partial^{2} \xi}{\partial x^{2}}=\frac{\partial^{2} \eta}{\partial x^{2}}=0,
$$

where $u$ is the longitudinal velocity component, $\xi$ and $\eta$ are the radial and angular components of vorticity $\boldsymbol{\Omega}=\operatorname{curl} \mathbf{u}$, where $\mathbf{u}=\{u, v, w\}$ is the flow velocity vector in cylindrical coordinates $x, r$ and $\theta$. Under these conditions a reflected wave apparently does not appear, or is very weak.

At the input cross-section of the pipe the longitudinal velocity component was assumed to take to the form of a Poiseuille profile $u_{0}\left(1-r^{2} / R^{2}\right)$, weakly disturbed by a harmonic force at the frequency $\omega=0.36 u_{0} / R$, i.e.,

$$
\begin{aligned}
& u=u_{0}\left(1-\frac{r^{2}}{R^{2}}\right)+A \operatorname{Re}\left(u^{\prime}(r) e^{-i \omega t}\right) \cos \theta \\
& v=A \operatorname{Re}\left(v^{\prime}(r) e^{-i \omega t}\right) \cos \theta, \quad w=A \operatorname{Re}\left(w^{\prime}(r) e^{-i \omega t}\right) \sin \theta,
\end{aligned}
$$

where $u^{\prime}(r), v^{\prime}(r)$ and $w^{\prime}(r)$ are the components of the Orr-Sommerfeld vector-eigenfunction at frequency $\omega, R$ is the pipe radius, and $A$ is the disturbance amplitude. The velocity $u_{0}$ and the pipe radius $R$ were set such that the Reynolds number $\operatorname{Re}$ was equal to 4000 . As the amplitude $A$ exceeded a certain critical value $(A \geq A$ cr $)$, 

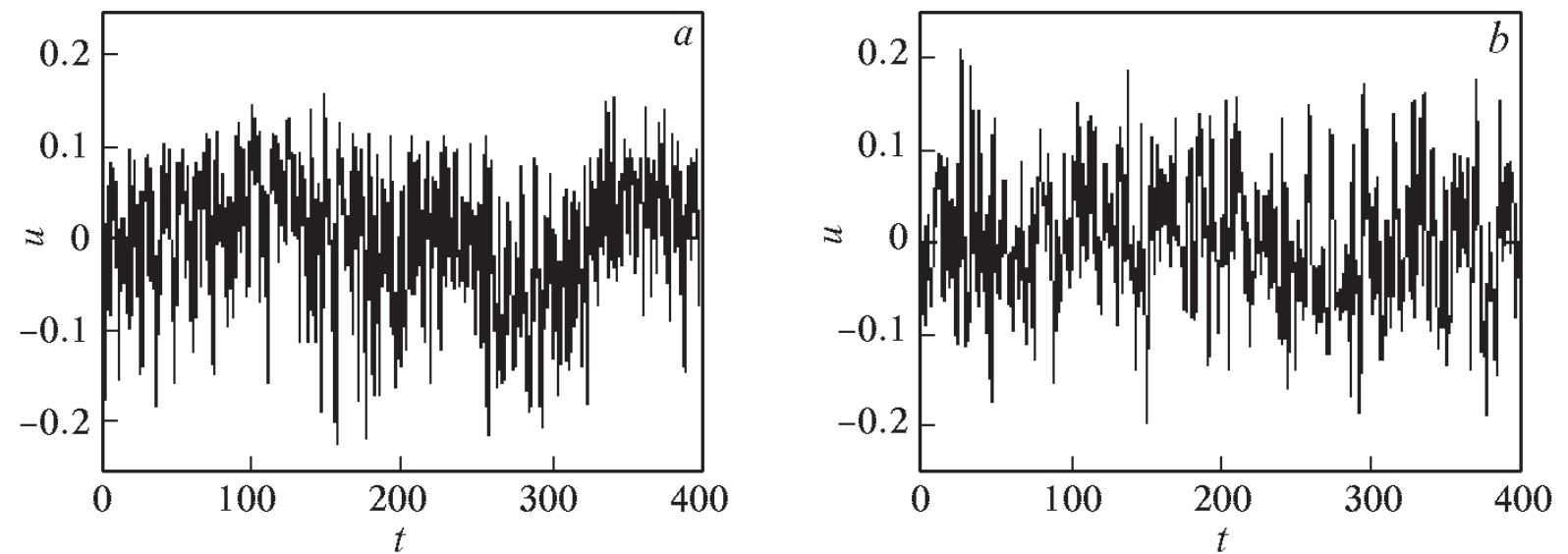

Figure 2. Results of numerical experiments on pipe flow. View of the turbulent velocity pulsations (a) with periodic boundary conditions and (b) with the boundary conditions (1), (2). After N.V. Nikotin. ${ }^{7}$

random high-frequency pulsations appeared in the flow after a short time interval. They occupied all the lower part of the pipe from $x=x_{0}$, where $x_{0}$ depended only weakly on the distance $r$ from the pipe axis. It turned out that the value of $x_{0}$ decreased as $A$ became larger. The appearance of turbulent pulsations was accompanied by corresponding deformation of the profile of the longitudinal constituent of the mean velocity: at the pipe axis the mean velocity decreased, whereas near the pipe wall it increased. The instantaneous distributions of the longitudinal velocity component in a steady regime for $A / u_{0}=0.04$ are shown in Fig. 1 . We note that a similar deformation of the mean velocity profile with increasing turbulent pulsations occurs in jet flows as well.

As the amplitude $A$ gradually decreased, the turbulent region drifted progressively downstream and disappeared at a certain value of $A$. It is known ${ }^{9}$ that Poiseuille flow in a circular pipe, in contrast to that in a plane channel, possesses the property that laminar flow is stable with respect to small perturbations for any Reynolds number. However, in the case of sufficiently large Reynolds numbers, such a flow is unstable with respect to finite perturbations. If an attractor existed corresponding to the turbulent mode, and if the role of the harmonic disturbance was to lead phase trajectories into the attractor basin, then turbulence should not disappear following cessation of the harmonic disturbance.

It may be inferred from Fig. 1 that the development of turbulence for $A \geq A_{\mathrm{cr}}$ is associated with a peculiar phase transition at the point $x=x_{0}$ induced by an amplification of the noise that is always present in any numerical experiment owing to rounding errors. The harmonic disturbance plays a dual role. First, it causes the appearance of instability and, secondly, it initiates the phase transition, much as occurs in a pendulum with a randomly vibrated suspension axis, ${ }^{10}$ or in jets under low-frequency acoustic forcing. ${ }^{11}$ It is no accident that the transition to turbulence was observed by Nikitin only for low-frequency disturbances (for Strouhal numbers of order 0.1).

Possible counter-arguments against the above ideas lie in the fact that numerical simulation results obtained with periodic boundary conditions are very close to those observed experimentally. But the data obtained by Nikitin in the numerical experiment described above are also close to numerical data for periodic boundary conditions. ${ }^{7}$ The visual similarity of turbulent pulsations calculated for periodic conditions, and for the boundary conditions (1), (2), is illustrated in Fig. 2. This similarity may be explained by the fact that many nonlinear oscillatory systems possess such pronounced intrinsic properties that they exhibit these properties independently of the means of excitation. Some examples of such (non-hydrodynamic) systems are described by Landa and Rabinovitch. ${ }^{12}$

Note that our discussion does not apply to so-called closed flows, e.g. to the Couette flow between two rotating cylinders or spheres. In closed flows there is always feedback linking the output of the amplifier to its input, so that they consequently become self-oscillatory. 

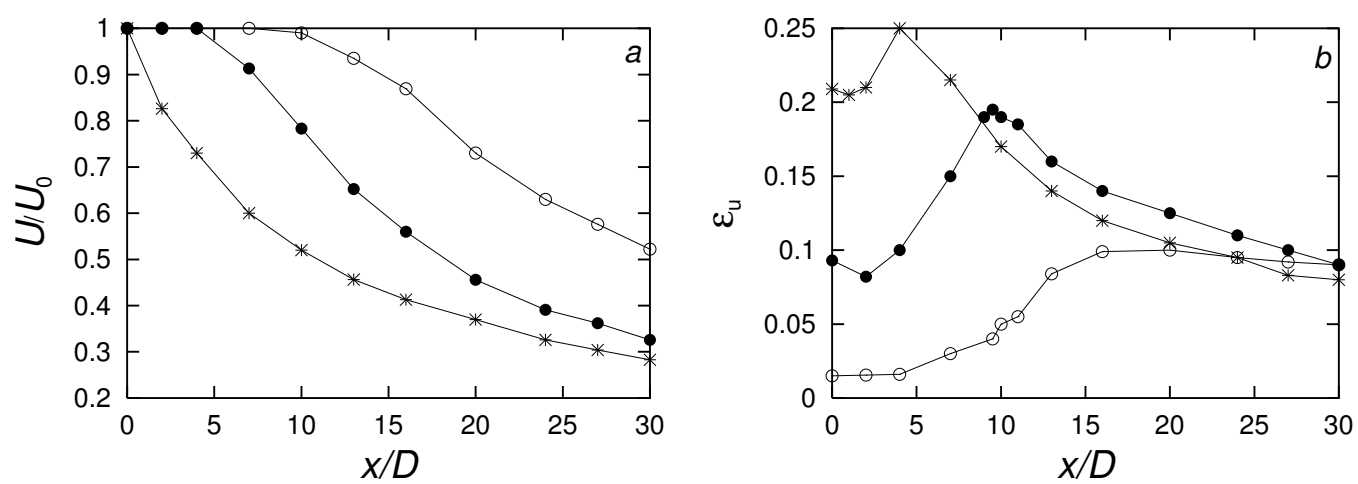

Figure 3. Experimental measurements on a submerged jet. The dependences of (a) the relative mean velocity $U / U_{0}$ and $(b)$ of the relative root-mean-square pulsation of the longitudinal component of hydrodynamic velocity $\epsilon_{u}=\sqrt{\overline{u^{2}}-U(0)^{2}} / U_{0}$ along the jet axis on the relative distance $x / D$ from the nozzle exit section, for three intensities of the disturbance at the nozzle exit section: $\epsilon_{u}(0)=0.015,0.0930 .209$ (curves marked by open circles, filled circles and stars, respectively). After Ginevsky et al. ${ }^{11}$

\section{A PHASE TRANSITION IN JET FLOWS}

A peculiar phase transition at a certain distance $x_{c r}$ from the nozzle, induced by an amplification of the input noise, is also observed experimentally in jet flows. It reveals itself both in the drastic decrease of the mean velocity and in the drastic increase of the turbulent pulsation intensity. The value of $x_{c r}$ is the smaller the larger is the noise intensity $\epsilon_{u}(0)$ at the nozzle exit section. This is illustrated in Fig. 3.

The phase transition can be controlled by harmonic acoustic forcing at an appropriate frequency. The value of $x_{c r}$ increases in the case of high-frequency forcing $\left(\mathrm{St}_{\mathrm{a}}>1.5\right)$ and decreases in the case of low-frequency forcing $\left(0.2<\mathrm{St}_{\mathrm{a}}<1.5\right)$, where $\mathrm{St}_{\mathrm{a}}=\omega_{\mathrm{a}} D / U_{0}$ is the Strouhal number corresponding to the acoustic wave of frequency $\omega_{\mathrm{a}}$ (see Fig. 4). Effects similar to those described above are also observed for other means of periodic forcing of the jet: e.g. longitudinal or radial vibration of the nozzle, or a pulsating rate of fluid outflow from the nozzle. $^{11}$
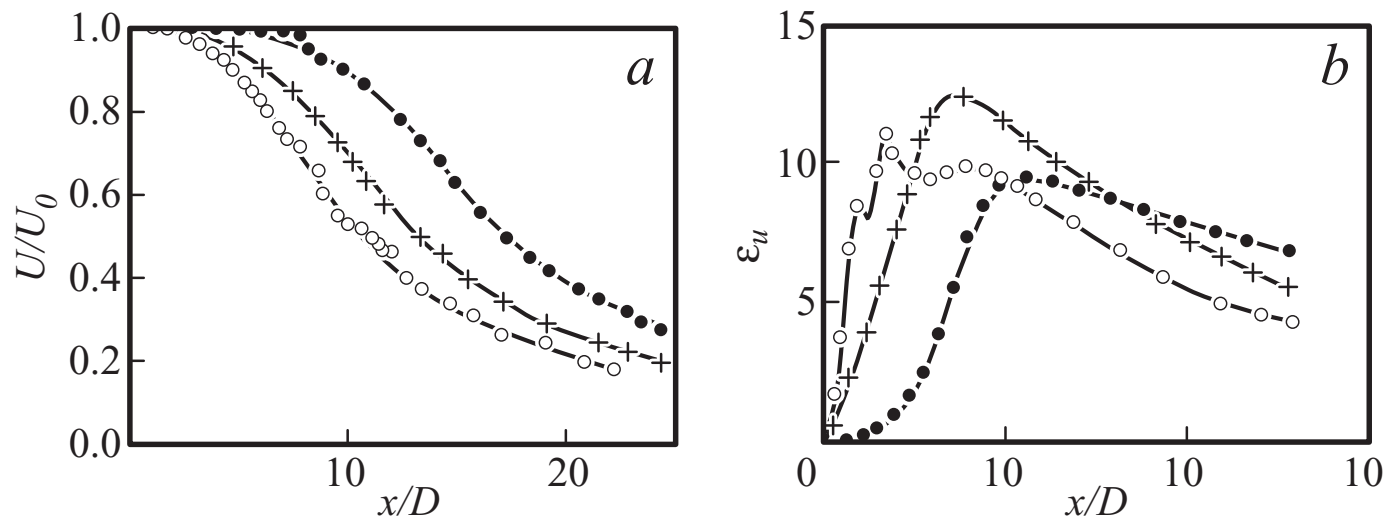

Figure 4. Experimental dependences: of (a) the relative mean flow velocity along the jet axis $U / U^{(0)}$ and $(b)$ of the relative root-mean-square pulsation of the longitudinal component of hydrodynamic velocity $\epsilon_{u}$ ) (in $\%$ ) on $x / D$ under a longitudinal acoustic forcing at $\mathrm{St}_{\mathrm{a}}=0.25$ (open circles), $\mathrm{St}_{\mathrm{a}}=2.75$ (filled circles). The crosses show what happens in the absence of forcing. The amplitude of the oscillatory velocity in the acoustical wave on the jet axis near the nozzle exit constitutes $0.07 \%$ of $U_{0}$. After Ginevsky et al. ${ }^{11}$ 

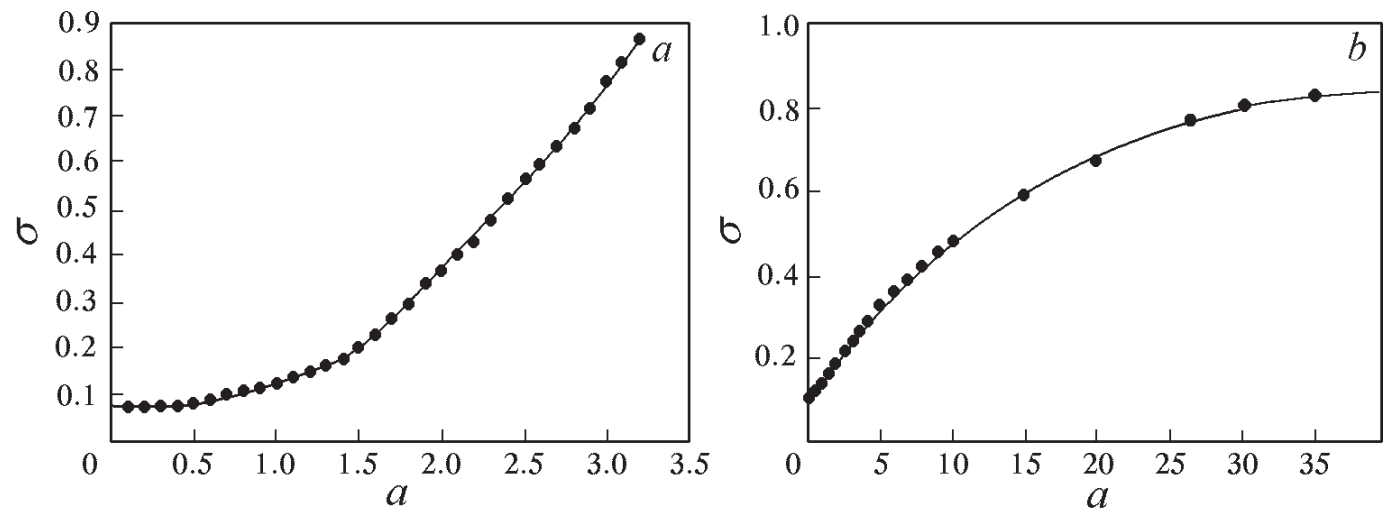

Figure 5. The calculated dependences of $\sigma$ on the amplitude $a$ of low-frequency vibration for $\omega_{0}=1, \beta=0.1, \alpha=100$ and: (a) $\kappa\left(2 \omega_{0}\right) / \kappa_{c r}=1.89, \omega_{a}=0.3 ;(b) \kappa\left(2 \omega_{0}\right) / \kappa_{c r}=2.23, \omega_{a}=1.5$.

\section{ANALOGY TO PENDULUM WITH RANDOMLY VIBRATED SUSPENSION AXIS}

The hypothesis that the onset of turbulence can usefully be considered as a noise-induced phase transition was first proposed by Landa, ${ }^{13}$ who studied the simplest physical system wherein such a transition is possible: the pendulum with a randomly vibrated suspension axis. ${ }^{10,14-17}$ It was found that profound parallels exist ${ }^{18-21}$ between $^{10}$ turbulent processes in nonclosed fluid flows and noise-induced oscillations in such a pendulum.

In the simplest case, when additive noise is neglected, the equation describing the oscillations of a pendulum with a randomly vibrated suspension axis is:

$$
\ddot{\varphi}+2 \beta\left(1+\alpha \dot{\varphi}^{2}\right) \dot{\varphi}+\omega_{0}^{2}(1+\xi(t)) \sin \varphi=0,
$$

where $\varphi$ is the pendulum's angular deviation from the equilibrium position, $2 \beta\left(1+\alpha \dot{\varphi}^{2}\right) \dot{\varphi}$ is proportional to the moment of the friction force which is assumed to be nonlinear, $\omega_{0}$ is the natural frequency of small oscillations, and $\xi(t)$ is a comparatively wide-band random process with nonzero power spectral density at the frequency $2 \omega_{0}$.

When the intensity of the suspension axis vibration, characterized by the spectral density of $\xi(t)$ at frequency $2 \omega_{0}\left(\kappa\left(2 \omega_{0}\right)\right)$, exceeds a certain critical value proportional to the friction factor $\beta$, excitation of pendulum oscillations occurs, and the variance of the pendulum's angular deviation becomes nonzero. It should be noted that close to the excitation threshold, the pendulum oscillations possess the property of so called on-off-intermittency. ${ }^{22}$

As described ${ }^{23}$ by Frisch and Morf, high-pass filtering of turbulent velocity pulsations reveals their intermittent behavior. This phenomenon was studied ${ }^{20}$ both for experimental velocity pulsations in a jet measured by Zaikin and also for the pendulum oscillations considered above. In each case it was observed on-off intermittency after high-pass filtering. This fact can be considered as an additional argument in support of the parallels between noise-induced pendulum oscillations and turbulent processes in jets.

It is important to note that the response of the pendulum to a small additional harmonic force (additional vibration of the suspension axis) is similar to the response of a jet to an acoustic force.

Thus, just as in the case of turbulent jets, the noise-induced pendulum oscillations under consideration can be controlled by a small additional harmonic force. Its inclusion can be effected by substitution into Eq. (3) of $\xi+a \cos \omega_{a} t$ in place of $\xi$, where $a$ and $\omega_{a}$ are respectively the amplitude and frequency of the additional vibration of the suspension axis. If the frequency of the additional forcing is relatively low, then this forcing intensifies the pendulum oscillations and lowers the excitation threshold; vice versa, a relatively high-frequency forcing suppresses the pendulum oscillations and increases the excitation threshold. The intensification of the pendulum oscillations by a low-frequency additional vibration is illustrated in Fig. 5 for two values of the vibration frequency. We see that the lower the forcing frequency is, the larger the variance of the oscillation becomes. Just as for jets, when the forcing amplitude becomes relatively large, the pendulum's oscillation amplitude saturates. 

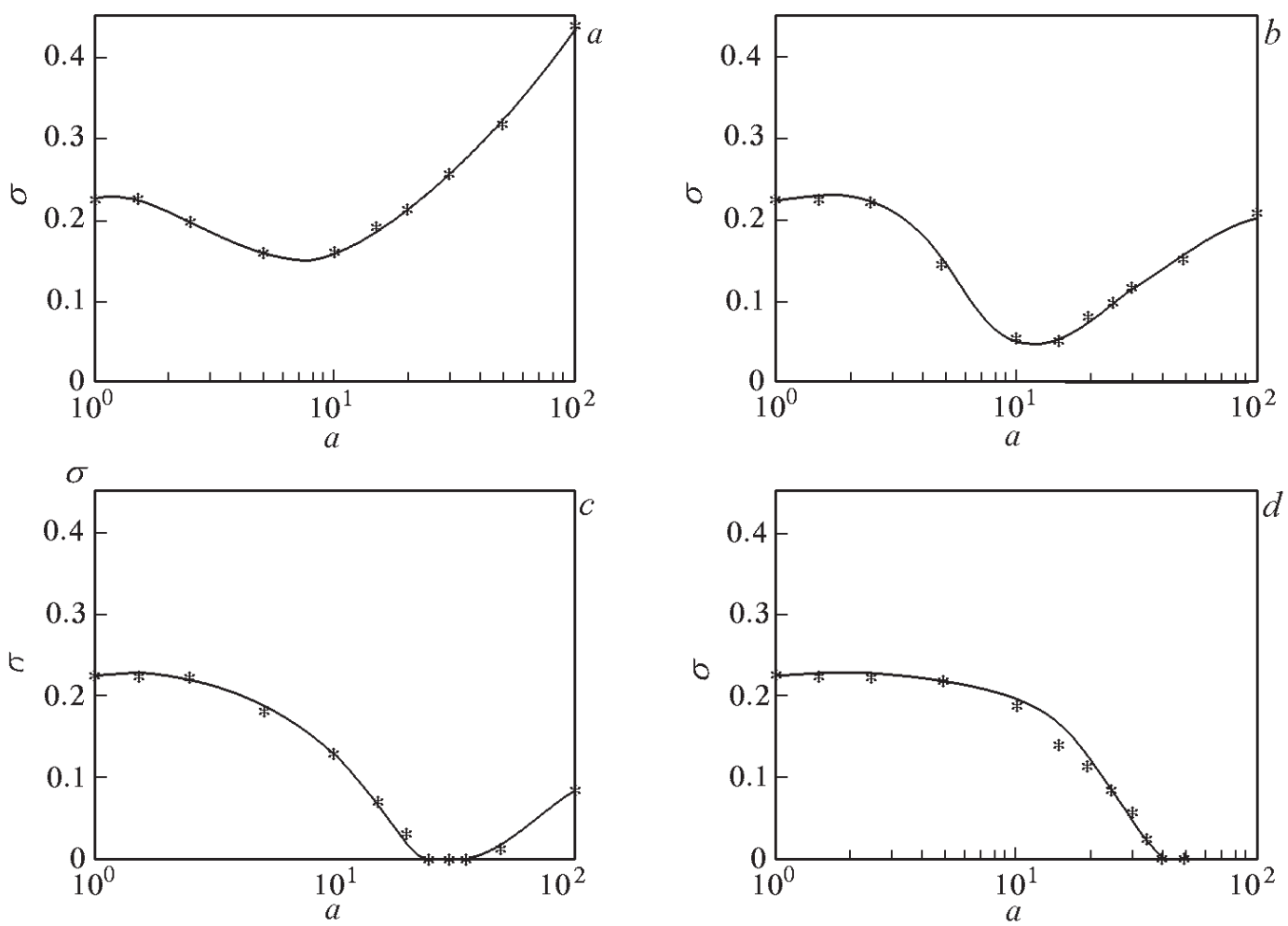

Figure 6. Numerical simulation showing the effect of a high frequency harmonic force $a \cos \omega t$ on the variance of the pendulum angle $\phi$. Results are shown for $\omega_{0}=1, \beta=0.1, \alpha=100, \kappa\left(2 \omega_{0}\right) / \kappa_{c r}=5.6$ and: (a) $\omega_{a}=3.5 ;(b) \omega_{a}=6 ;(c)$ $\omega_{a}=11 ;(d) \omega_{a}=19.757$.

Below we consider the possibility of suppressing noise-induced pendulum oscillations by the addition of a high-frequency vibration. Numerical simulation of Eq. (3) with $\xi+a \cos \omega_{a} t$ in place of $\xi$, where $\omega_{a}>2$, shows that such suppression can occur. The results of the simulation are illustrated in Fig. 6, where the dependences of the variance of the angle $\varphi$ on $a$ for a number of values of the vibration frequency are shown. It is evident from this figure that, for small amplitudes of the high-frequency vibration, this vibration has little or no effect on the noise-induced oscillations. As the amplitude increases, however, the intensity of the noise-induced oscillations decreases rapidly. When the amplitude exceeds a certain critical value (for $\omega_{a}=19.757$ it is equal to 42 ) the oscillations are suppressed entirely. As the amplitude increases further the oscillations reappear, but now because the conditions required for parametric resonance come into play. For smaller frequencies $\omega_{a}$, the behavior of the pendulum oscillations is different. It is seen that the variance of $\varphi$ at first decreases, passes through a certain minimum value, and then increases again. It is important to note that this minimum value becomes smaller with increasing forcing frequency, but that it is attained for larger forcing amplitudes at higher frequencies. The dependence shown in Fig. 6 a closely resembles the corresponding dependences for a jet presented in Fig. 7 .

The dependences of $\sigma$ on $\omega_{a}$ for a number of fixed amplitudes of the additional vibration are illustrated in Fig. 8. Again, these dependences closely resemble the corresponding ones for a jet shown in Fig. 9.

The presence of a small additive noise, in addition to the multiplicative one in Eq. (3), does not change the behavior of the pendulum qualitatively, but it gives rise to large quantitative differences. The principal one is the impossibility of achieving full suppression of the pendulum oscillations. Nevertheless, a very marked attenuation of the oscillation intensity occurs. It should be emphasized that, in the case of turbulence, full suppression is of course impossible. 

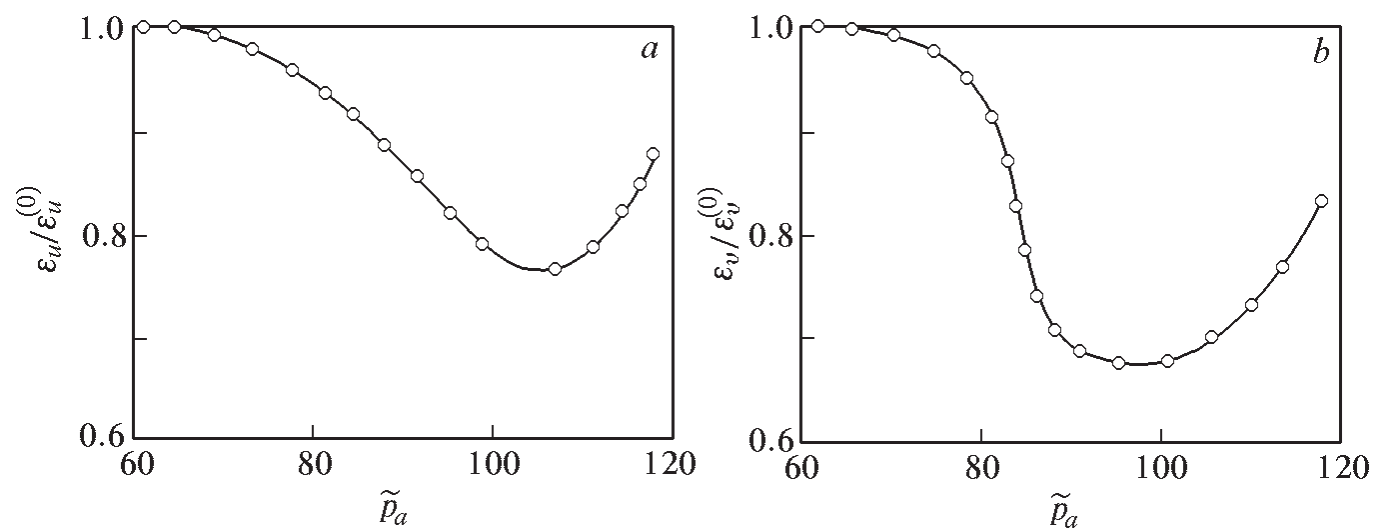

Figure 7. The experimental dependences of the relative root-mean-square pulsation of the suppression factors (a) $\epsilon_{u} / \epsilon_{u}^{(0)}$ and (b) $\epsilon_{v} / \epsilon_{v}^{(0)}$ of the longitudinal and radial components of hydrodynamical velocity on the relative amplitude of acoustic pressure $\tilde{p}_{\mathrm{a}}$ measured in decibels, for $\mathrm{St}_{\mathrm{a}}=2.35, x / D=8 ; \epsilon_{u}^{(0)}$ and $\epsilon_{v}^{(0)}$ are relative pulsations of the longitudinal and radial velocity components in the absence of acoustic excitation. After Ginevsky et al. ${ }^{11}$

\section{APPLICATION OF KRYLOV-BOGOLYUBOV METHOD}

We have studied the onset of turbulence in subsonic submerged jets analytically by using the asymptotic KrylovBogolyubov method. ${ }^{25}$ This is possible when the intensity of the input noise is sufficiently small that the turbulent processes near the nozzle can be described by linearized Navier-Stokes equations. Apparently, for real jets the intensity of the input noise is much more than that is necessary for the validity of our theory. It is possible that such small input noise is present in jet flows of superfluid helium. ${ }^{26}$ Nevertheless, explicit consideration of the amplification of the input noise allows us to account for many known experimental results within the initial part of a jet and find a clearly defined phase transition. Moreover, it follows from our theory that the commonly accepted explanation for the well-known shift of velocity pulsation power spectra towards the low-frequency region is in fact erroneous. According to this explanation, the shift of the power spectra occurs because of feedback via an acoustic wave generated where vortex pairing occurs, as seen in experiments. We will show that the reason for the spectral shift lies in the jet's divergence; and that this shift causes the increase of spatial scale

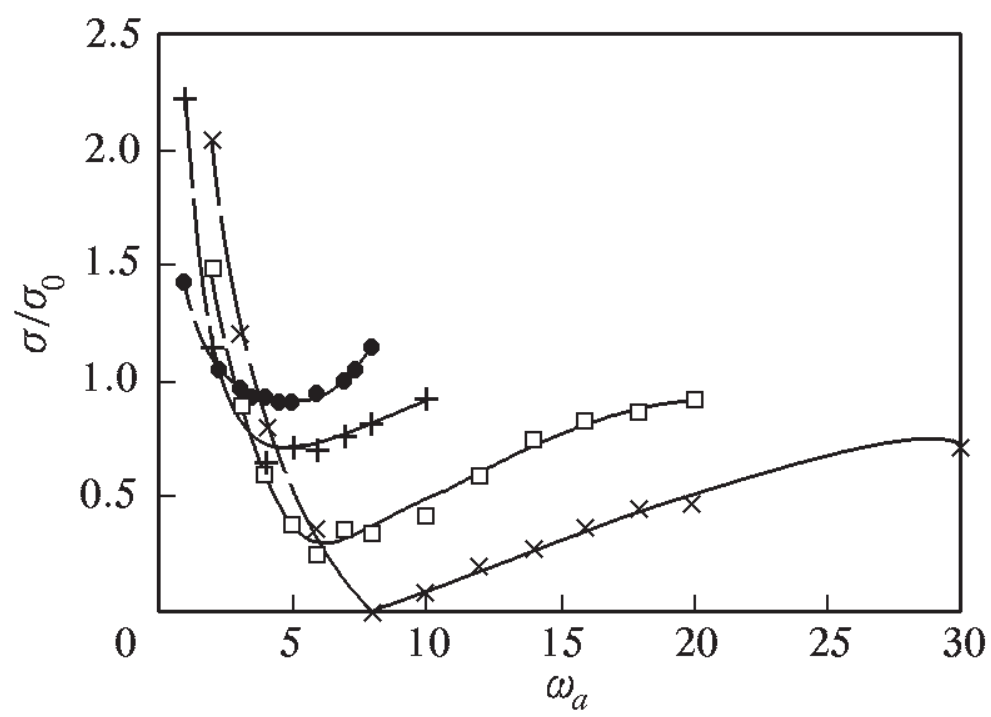

Figure 8. Numerical simulation of the pendulum with randomly vibrated suspension axis, showing the dependence on $\omega_{a}$ of $\sigma / \sigma_{0}$, where $\sigma_{0}$ is the value of $\sigma$ in the absence of additional vibration. Results are plotted for $\kappa\left(2 \omega_{0}\right) / \kappa_{c r}=5.6$, $a=2.5$ (filled circles), $a=5$ (pluses), $a=10$ (squares), and $a=20$ (crosses). 


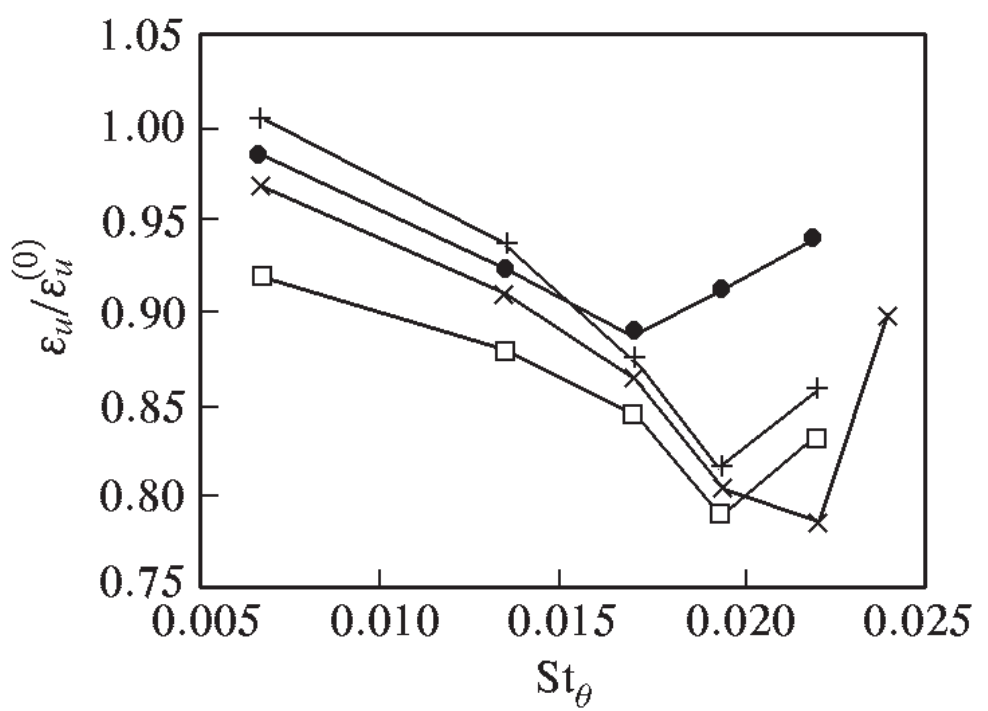

Figure 9. The experimental dependences on the Strouhal number $\mathrm{St}_{\theta}=(\theta / D) \mathrm{St}$ of the suppression factor $\epsilon_{u} / \epsilon_{u}^{(0)}$, where $\epsilon_{u}^{(0)}$ is the relative intensity of the longitudinal velocity pulsations in the absence of acoustic forcing, for $x / \theta=200$. The plots are constructed for four values of the amplitude of the oscillatory velocity in the acoustic wave, namely $0.5 \%$ of $U_{0}$ (circles), $2.5 \%$ (pluses), $3.5 \%$ (crosses) and $4.5 \%$ (squares). After Nallasamy and Hussain. ${ }^{24}$

with increasing distance from the nozzle, and results in the observed vortex pairing. It is also shown that, as turbulence develops, its longitudinal and transverse scales increase. Our results coincide qualitatively and also, in specific cases, quantitatively, with known experimental data.

We consider a plane jet issuing from a nozzle of width $2 d$. Neglecting compressibility, we may describe the processes in such a jet by the two-dimensional Navier-Stokes equations for the stream function $\Psi(t, x, y)$ and the vorticity $\tilde{\Omega}(t, x, y)$. In dimensionless coordinates these equations are

$$
\begin{aligned}
& \tilde{\Omega}(t, x, y)=\Delta \Psi(t, x, y), \\
& \frac{\partial \tilde{\Omega}(t, x, y)}{\partial t}-\frac{\partial \Psi(t, x, y)}{\partial x} \frac{\partial \tilde{\Omega}(t, x, y)}{\partial y}+\frac{\partial \Psi(t, x, y)}{\partial y} \frac{\partial \tilde{\Omega}(t, x, y)}{\partial x}-\frac{2}{\operatorname{Re}} \Delta \tilde{\Omega}(t, x, y)=0,
\end{aligned}
$$
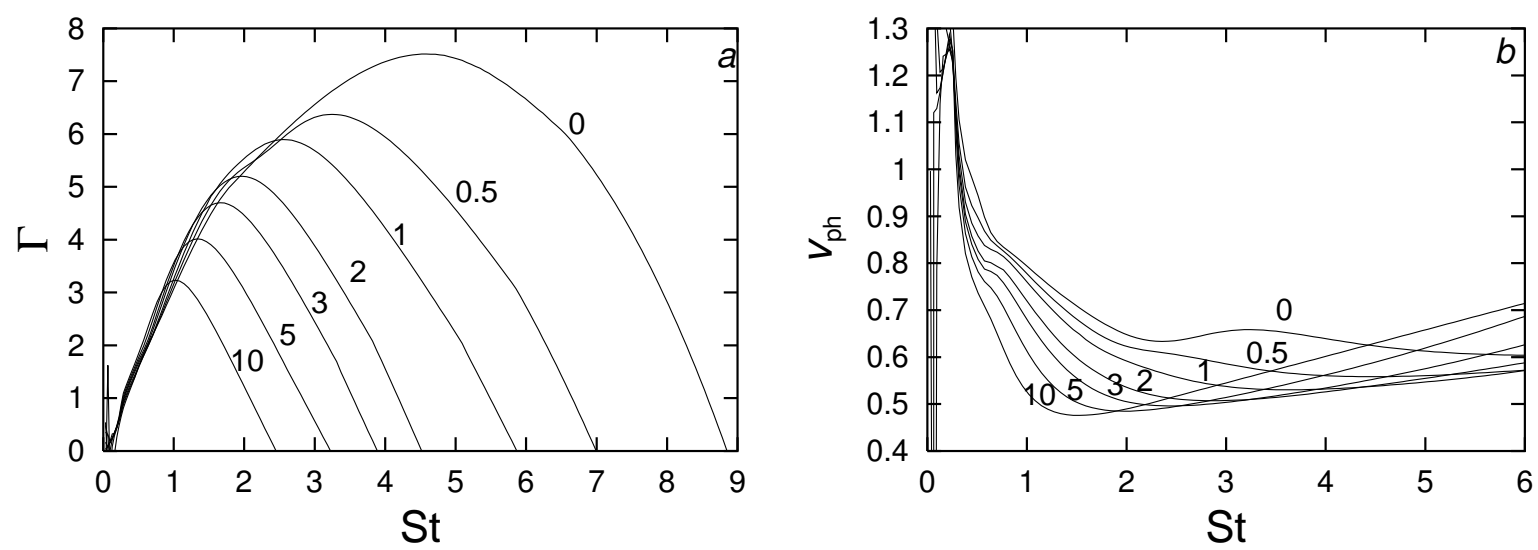

Figure 10. Results of a calculation based on the Krylov-Bogolyubov method. The dependences on the Strouhal number St for different $x$ of: (a) $\Gamma$ and (b) the wave phase velocity $v_{\mathrm{ph}}$ are shown for the values of $x$ indicated near the corresponding curve in each case. 
where $\Delta=\frac{\partial^{2}}{\partial x^{2}}+\frac{\partial^{2}}{\partial y^{2}}$ is the Laplacian, $\operatorname{Re}=2 U_{0} d / \nu$ is the Reynolds number, $\nu$ is the kinematic viscosity, $U_{0}$ is the mean flow velocity in the nozzle center, $x$ is the coordinate along the jet axis, and $y$ is the transverse coordinate. It should be noted that in so deciding on a dimensionless time, the circular frequencies $\omega=2 \pi f$ are measured in units of $\mathrm{S}=\omega d / U_{0} \equiv \pi \mathrm{St}$, where $\mathrm{St}=2 f d / U_{0}$ is the Strouhal number.

In accordance with the ideas presented above, the onset of turbulence is caused by random disturbances (noise) in the nozzle exit section. The authors of works ${ }^{27,28}$ devoted to the stability of jet flows mostly split the solution into mean values and small random disturbances. In our opinion this procedure is inappropriate for two reasons: first, exact equations for the mean values are unknown; and, secondly, the random disturbances make a significant contribution to the mean values. That is why we split the solution of Eqs. (4), (5) into dynamical and stochastic constituents. The dynamical constituents are described by stationary Navier-Stokes equations and differ from the mean values of the corresponding variables because of the quadratic nonlinearity.

We consider first the dynamical constituents of the velocity and vorticity. It follows from Eqs. (4), (5) that the dynamical constituents $\left.u_{\mathrm{d}}(x, y), v_{\mathrm{d}}(x, y)\right)$ and $\Omega_{\mathrm{d}}(x, y)$ are described by the equations

$$
\begin{aligned}
& \Omega_{\mathrm{d}}(x, y)=\frac{\partial u_{\mathrm{d}}(x, y)}{\partial y}-\frac{\partial v_{\mathrm{d}}(x, y)}{\partial x}, \\
& \frac{\partial u_{\mathrm{d}}(x, y)}{\partial x}+\frac{\partial v_{\mathrm{d}}(x, y)}{\partial y}=0, \\
& u_{\mathrm{d}}(x, y) \frac{\partial \Omega_{\mathrm{d}}(x, y)}{\partial x}+v_{\mathrm{d}}(x, y) \frac{\partial \Omega_{\mathrm{d}}(x, y)}{\partial y}-\frac{2}{\operatorname{Re}}\left(\frac{\partial^{2} \Omega_{\mathrm{d}}(x, y)}{\partial x^{2}}+\frac{\partial^{2} \Omega_{\mathrm{d}}(x, y)}{\partial y^{2}}\right)=0 .
\end{aligned}
$$

It is very difficult, if not impossible, to solve these nonlinear equations exactly. Therefore we choose $u_{\mathrm{d}}(x, y)$ in the form of a given function of $y$ with unknown parameters depending on $x$. The shape of this function must depend on whether the outflow from the nozzle is laminar or turbulent. For simplicity, we restrict our consideration to laminar nozzle flow. In this case we can set $u_{\mathrm{d}}(x, y)$ so that, at the nozzle exit section, the boundary layer is close in form to that described by the Blasius equation. ${ }^{29}$ Taking account of the entrainment of the ambient fluid, we set the velocity profile close to the Blasius one for $x=0$ in the form

$$
u_{\mathrm{d}}(x, y)=\frac{1}{1+\tanh \left(q / \delta_{00}+r_{0}\right)}\left[1-\tanh \left(q \frac{|y|-1}{\delta_{0}(x)}-r(x)\right)\right],
$$

where $q=3, \delta_{0}(x)$ and $r(x)$ are unknown functions of $x$, and $\delta_{0}(x)$ is the boundary layer thickness which is equal to $\delta_{00}$ for $x=0, r_{0}=r(0)$. To find the unknown functions in the expression (9), we use the conservation laws for the fluxes of momentum and energy which we derive starting from Eqs. (6)-(8). As a result we find $r(x) \approx r_{0}=0.5$ and $\delta_{0}(x)=\sqrt{\delta_{00}^{2}+2 k x}$, where $k=16 q^{2} /(3 \operatorname{Re})$.

We will refer to deviations from the dynamical constituents as stochastic components. To study the jet stability we linearize Eqs. (4), (5) relative to these components. It should be noted that finding a solution of the linearized equations for the stochastic components is similar to the well known problem of the linear instability of a jet flow and reduced to a non-self-adjoint boundary-value problem with zero boundary conditions at $y= \pm \infty$. The solution of this boundary-value problem allows us to find the gain factor $\Gamma$ and wave phase velocity $v_{\mathrm{ph}}$, and their dependences on the Strouhal number St. The calculated values of $\Gamma$ and $v_{\mathrm{ph}}$ as functions of St are shown for different $x$ in Fig. 10 a and $b$. The resonant character of the dependences of the gain factor on the Strouhal number that we have found indicates that each jet cross-section can be considered as an oscillator whose natural frequency decreases with increasing distance from the nozzle. This fact justifies consideration of a jet as a chain of coupled resonant amplifiers, which in turn allows us to understand the analogy between noise-induced pendulum oscillations and the turbulent processes in a jet.

By using the first and second approximations of the Krylov-Bogolyubov method we have calculated the change of the mean velocity against the longitudinal and transversal coordinates and the variance of the longitudinal velocity pulsations. These calculations allow us to follow the occurrence of the phase transition. The plots of $\epsilon_{u}$ versus $x$ are presented in Fig. 11 for two values of $y(y=0$ and $y=0.7)$. It is seen that the found dependences 

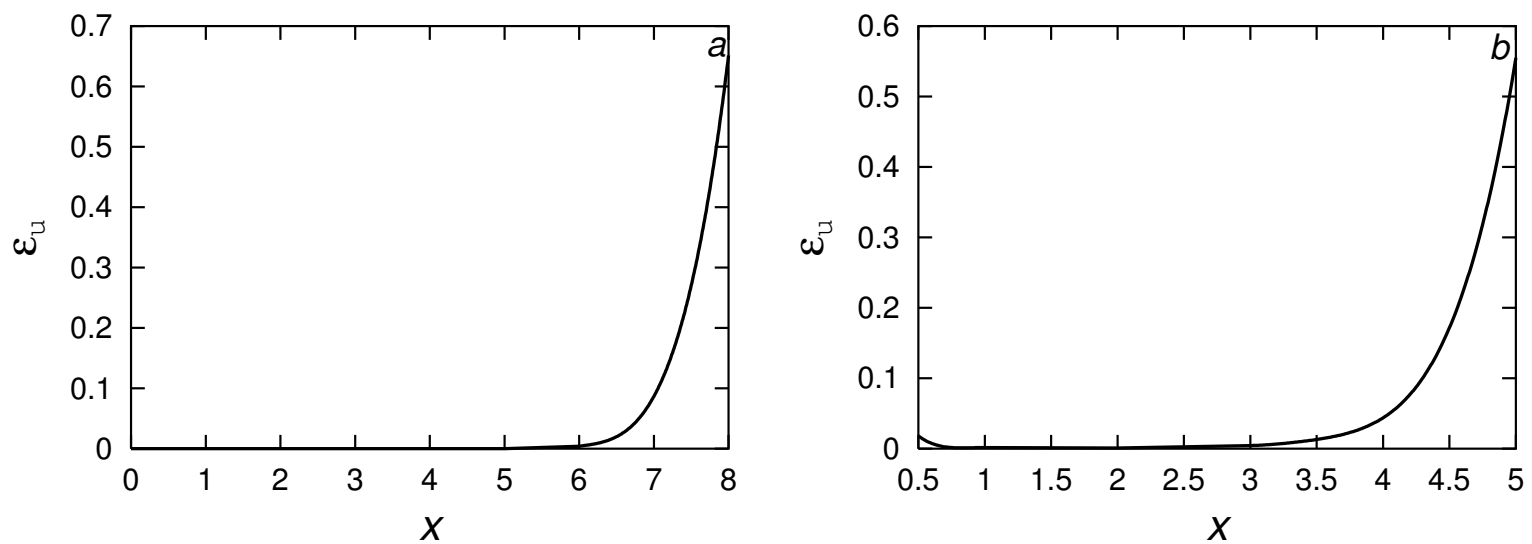

Figure 11. Plots of calculated mean-root-square values of turbulent velocity pulsations versus $x$ for $(a) y=0$ and $(b)$ $y=0.7$.

of $\epsilon_{u}$ on $x$ closely resemble the dependence of the variance of noise-induced pendulum oscillations on the noise intensity in the case of small additive noise. As would be expected, at the jet axis the phase transition occurs for larger $x$ than for $y=0.7$.

The increase of $\epsilon_{u}$ with increasing $x$ is correlated with the decrease of the mean velocity (see Fig. 12), but these changes are not exactly equivalent.

\section{CONCLUSIONS}

The theoretical approach described above enables us to account for many experimental results, and to demonstrate that some widely-accepted interpretations are in fact erroneous. It has led us to a somewhat different and, we believe, more realistic perspective. In particular, we conclude that -

1. The shift in the velocity pulsation power spectra towards the low-frequency domain is caused mainly by the jet divergence, and not by pairing of vortices. It can therefore be calculated within the linear approximation.

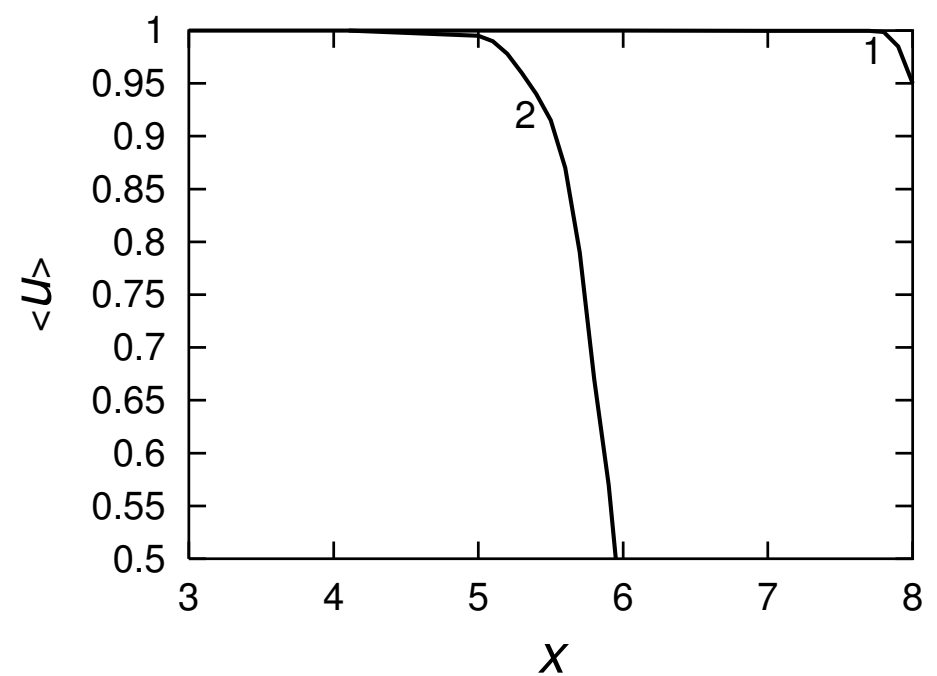

Figure 12. Calculated dependences of the mean velocity $\langle u(x, y)\rangle$, taking account of the correction caused by the turbulent pulsations on $x$ for (1) $y=0$ and (2) $y=0.7$. 
2. The transformation of the mean velocity profile, and the onset of the phase transition, can be found without any need to use the concept of turbulent viscosity.

3. Our quasi-linear theory is valid only for small intensities of the random disturbance (noise); but the noise intensity at the nozzle exit needed to maintain the turbulence may, correspondingly, also be very small. For larger intensities, the development of turbulence is from the very outset an essentially nonlinear process.

4. The change in the variance of the velocity pulsation with distance from the nozzle, like the change in the angular variance with increasing noise intensity in the pendulum with a randomly vibrated suspension axis, closely resembles changes in the order parameter with increasing temperature for a second order phase transition. This is the basis for our conjecture that these phenomena can appropriately be considered as noise-induced non-equilibrium phase transitions.

\section{Acknowledgements}

It is a pleasure to acknowledge useful discussions with G.V. Kolmakov and helpful advice related to computation from A.N. Sil'chenko and I.Kh. Kaufman. The research was supported in part by the Engineering and Physical Sciences Research Council (UK), and by the Royal Society of London to whom PSL is indebted for a visiting research fellowship at Lancaster during which much of the review was completed.

\section{REFERENCES}

1. P.D. Drazin and W.H. Reid, Hydrodynamic Stability, (Cambridge Univ. Press, Cambridge, 1981).

2. L.D. Landau, On the turbulence problem, DAN SSSR 44 (1944) 339-342 (in Russian).

3. D. Ruelle and F. Takens, On the nature of turbulence, Comm. Math. Phys. 20 (1971) 167-192.

4. D. Ruelle D. Strange attractors as a mathematical explanation of turbulence, Lect. Notes in Phys., Statistical Models and Turbulence 12 (1975) 292-316.

5. Yu.I. Neimark and P.S. Landa, Stochastic and Chaotic Oscillations, (Kluwer Academic Publishers, Dordrecht, 1992).

6. Yu.L. Klimontovich, Turbulent Motion and the Structures of Chaos, (Kluwer Academic Publ., Dordrecht, 1991).

7. N.V. Nikitin, Direct numerical simulation of three-dimensional turbulent flows in circular pipes, Izv. RAN, MZhG (1994) No 6 14-26.

8. N.V. Nikitin, Spatial approach to numerical simulation of turbulence in pipes, DAN 343(1995) 767-770.

9. H. Salwen and C.E. Grosch, The stability of Poiseuille flow in a pipe of circular cross-section, J. Fluid Mech. 54 (1971) 93-112.

10. P.S. Landa, A.A. Zaikin, M.G. Rosenblum, and J. Kurths, Control of noise-induced oscillations of a pendulum with a randomly vibrated suspension axis, Phys. Rev. E 56 (1997) 1465-1470.

11. A.E. Ginevsky, Ye.V. Vlasov and R.K. Karavosov, Acoustic Control of Turbulence Jets, (Springer, Heidelberg, 2004).

12. P.S. Landa and A. Rabinovitch, Exhibition of intrinsic properties of certain systems in response to external disturbances. Phys. Rev. E 61 (2000) 1829-1838.

13. P.S. Landa, Turbulence in nonclosed fluid flows as a noise-induced phase transition, Europhys. Lett. 36 (1996) 401-406.

14. P.S. Landa and A.A. Zaikin, Noise-induced phase transitions in a pendulum with a randomly vibrated suspension axis, Phys. Rev. E 54 (1996) 3535-3544.

15. P.S. Landa, Nonlinear Oscillations and Waves in Dynamical Systems (Kluwer Academic Publ., Dordrecht, 1996).

16. P.S. Landa and A.A. Zaikin, Noise-induced phase transitions in nonlinear oscillators, in: AIP Conference Proceedings 465 (Computing Anticipatory Systems, CASYS'98, Liege, Belgium 1998), 419-433.

17. P.S. Landa, A.A. Zaikin and J. Kurths, On noise-induced transitions in nonlinear oscillators, in: Stochastic Processes in Physics, Chemistry, and Biology, ed. by J.A. Freynd, T. Pöschel, (Springer, Berlin, 2000), 268-279. 
18. P.S. Landa, Universality of oscillation theory laws. Types and role of mathematical models, Discrete Dynamics in Nature and Society 1 (1997) 99-110.

19. P.S. Landa, Onset of turbulence in open liquid flows as a nonequilibrium noise-induced second-order phase transition, Techn. Physics 43 (1998) 27-34.

20. P.S. Landa, A.S. Ginevsky, Ye.V. Vlasov, and A.A. Zaikin, Turbulence and coherent structures in subsonic submerged jets. Control of the Turbulence, Int. J. Bifurcations and Chaos 9 (1999) 397-414.

21. Ye.V. Vlasov et al., On analogy between the responce on periodical excitation of a turbulent jet and the pendulum with randomly vibrated suspension axis, J. Eng. Phys. and Thermophys. 75 (2002) No 4, 90-93.

22. P.S. Landa, A.A. Zaikin, M.G. Rosenblum, and J. Kurths, On-off intermittency phenomena in a pendulum with a randomly vibrated suspension axis, Chaos, Solitons and Fractals 9 (1998) 157-169.

23. U. Frisch and R. Morf, Intermittency in nonlinear dynamics and singularities at complex times, Phys. Rev. A 23 (1981) 2673-2705.

24. M. Nallasamy and A.K.M.F. Hussain, Effects of excitation on turbulence levels in a shear layer, Trans. ASME 111 (1989) 102-104.

25. P.S. Landa and P.V.E. McClintock, Development of turbulence in subsonic submerged jets, submitted to Physics Reports.

26. High Reynolds Number Flows Using Liquid and Gaseous Helium. Discussion of Liquid and Gaseous Helium as a Test Fluid, ed. by R.J. Donnelly, (Springer-Verlag, New York, 1991).

27. D.G. Crighton and M. Gaster, Stability of slowly diverging jet flow, J. Fluid Mech. 77 (1976) 397-413.

28. A. Michalke, Survey on jet instability theory, Progr. Aerosp. Sci. 21 (1984) 159-199.

29. H. Schlichting, Grenzschicht-Theorie (Verlag G. Braun, Karlsruhe, 1965). 